\title{
SMALL-TIME ASYMPTOTICS OF OPTION PRICES AND FIRST ABSOLUTE MOMENTS
}

\author{
JOHANNES MUHLE-KARBE, ${ }^{*}$ Universität Wien \\ MARCEL NUTZ, ${ }^{* *}$ ETH Zürich
}

\begin{abstract}
We study the leading term in the small-time asymptotics of at-the-money call option prices when the stock price process $S$ follows a general martingale. This is equivalent to studying the first centered absolute moment of $S$. We show that if $S$ has a continuous part, the leading term is of order $\sqrt{T}$ in time $T$ and depends only on the initial value of the volatility. Furthermore, the term is linear in $T$ if and only if $S$ is of finite variation. The leading terms for pure-jump processes with infinite variation are between these two cases; we obtain their exact form for stable-like small jumps. To derive these results, we use a natural approximation of $S$ so that calculations are necessary only for the class of Lévy processes.
\end{abstract}

Keywords: Option price; absolute moment; small-time asymptotics; approximation by Lévy processes

2010 Mathematics Subject Classification: Primary 91B25

Secondary 60G44

\section{Introduction}

We consider the problem of option pricing in mathematical finance where the price of an option on a stock $S$ is calculated as the expectation under a risk-neutral measure. As usual, we assume that the stock price is modeled directly under this measure and set the interest rate to zero. Therefore, our basic model consists of a càdlàg martingale $S$ on a filtered probability space $\left(\Omega, \mathcal{F},\left(\mathcal{F}_{t}\right)_{t \geq 0}, \mathrm{P}\right)$ satisfying the usual hypotheses; we assume for simplicity that $\mathcal{F}_{0}$ is trivial P-almost surely (P-a.s.). Our main interest concerns the small-time asymptotics of European call option prices,

$$
\mathrm{E}\left[\left(S_{T}-K\right)^{+}\right] \text {for } T \downarrow 0,
$$

where $x^{+}:=\max \{x, 0\}$ and $K \in \mathbb{R}$ is the strike price of the option. More precisely, we will mostly be interested in the leading-order asymptotics for the at-the-money case $K=S_{0}$. Option price asymptotics are used in finance to find initial values for model calibration procedures and also for model testing, as explained below. From a probabilistic point of view we can note that, up to a factor two, these are also the asymptotics of the centered absolute first moment and, at least for continuous $S$, of the expected local time at the origin.

In applications $S$ is often specified via a stochastic differential equation driven by a Brownian motion $W$ and a Poisson random measure $N(\mathrm{~d} t, \mathrm{~d} x)$ with compensator $F(\mathrm{~d} x) \mathrm{d} t$; i.e. $S$ is of

Received 6 July 2010; revision received 12 June 2011.

* Current address: Department of Mathematics, ETH Zürich, Rämistrasse 101, 8092 Zürich, Switzerland.

Email address: johannes.muhle-karbe@math.ethz.ch

** Current address: Department of Mathematics, Columbia University, 2990 Broadway, New York, NY 10027, USA.

Email address: mnutz@math.columbia.edu 
the form

$$
S=S_{0}+\sigma \cdot W+\kappa(x) *(N-F(\mathrm{~d} x) \mathrm{d} t),
$$

where $\sigma=\left(\sigma_{t}\right)$ and $\kappa=\left(\kappa_{t}(x)\right)$ are predictable integrands, and $\sigma \cdot W_{u}=\int_{0}^{u} \sigma_{t} \mathrm{~d} W_{t}$ as well as $\kappa(x) *(N-F(\mathrm{~d} x) \mathrm{d} t)_{u}$ denote the Itô integral and the integral $\int_{0}^{u} \int_{\mathbb{R}} \kappa_{t}(x)(N(\mathrm{~d} t, \mathrm{~d} x)-F(\mathrm{~d} x) \mathrm{d} t)$ of random measures, respectively. In view of the applications, we will adopt this representation for $S$, but we recall in Remark A.1 that this entails no essential loss of generality: every càdlàg martingale with absolutely continuous characteristics can be represented in the form (1.2), and this includes all models of interest.

The main results for the at-the-money asymptotics (1.1) are obtained under certain rightcontinuity conditions on the mapping $t \mapsto\left(\sigma_{t}, \kappa_{t}\right)$. If we exclude the trivial case $S \equiv S_{0}$, the possible convergence rates range from $\sqrt{T}$ to $T$. If $\sigma_{0} \neq 0$, i.e. in the presence of a Brownian component, the leading term is given by $\left(\left|\sigma_{0}\right| / \sqrt{2 \pi}\right) \sqrt{T}$ irrespective of the jumps. On the other hand, the leading term is $C T$ if and only if $S$ is of finite variation, and then $C$ is given explicitly in terms of $\kappa_{0}$ and $F$. For pure-jump processes with infinite variation, the rate may be anywhere between $\sqrt{T}$ and $T$, and it need not be a power of $T$. We consider a class of processes, containing most relevant examples, whose small jumps resemble those of an $\alpha$-stable Lévy process, where $\alpha \in[1,2)$. For $\alpha>1$, the leading term is $C T^{1 / \alpha}$, while, for $\alpha=1$, we find $C T|\log T|$; the constants are given explicitly.

The basic idea to obtain these results is to calculate the option price asymptotics for a simple model $Z$ which approximates $S$ in a suitable sense. More precisely, we obtain a natural approximation by freezing the coefficients $\sigma$ and $\kappa$ in (1.2) at time $t=0$, namely,

$$
Z=S_{0}+\sigma_{0} W+\kappa_{0}(x) *(N-F(\mathrm{~d} x) \mathrm{d} t) .
$$

Note that $\sigma_{0}$ and $\kappa_{0}(x)$ are deterministic since $\mathcal{F}_{0}$ is trivial. We show that the Lévy process $Z$ has the same leading-order asymptotics as $S$ under mild regularity conditions. Therefore, an explicit treatment is necessary only in the Lévy case, for which much 'finer' arguments are possible. We prove that we can pass, with an error of order $O(T)$, from one pure-jump Lévy process to another when the small jumps have a similar behavior and use this to reduce even further to very particular Lévy processes.

\subsection{Literature}

Owing to their importance for model calibration and testing, small-time asymptotics of option prices have received considerable attention in recent years; see [2], [5], [6], [7], [10], [11], [12], [13], [14], [15], [16], [17], [22], and [24]. A survey of recent literature is given in the introduction of Forde et al. [16]. We will review only the works most closely related to our study; in particular, we focus on the at-the-money case. Here Carr and Wu [7] is an early reference with results in the spirit of ours. The authors obtained by partially heuristic arguments that the order of convergence for finite-variation jumps is $O(\sqrt{T})$ in the presence of a Brownian component and $O(T)$ otherwise. This is in a general model including, e.g. exponential Lévy processes; however, a boundedness assumption on the coefficients of the log price excludes the application to the Heston model, for example. For the pure-jump case with infinite variation, the authors mentioned that there is a range of possibilities for the order of convergence and they illustrated this by the so-called log-stable model. Given option price data, the results are used to study whether the underlying process has jumps.

Durrleman [10] determined the rate $O(\sqrt{T})$ and the corresponding coefficient in a similar model, again with bounded coefficients and finite-variation jumps. The result is stated in terms of the implied volatility, which is an alternative parametrization for option prices (see also 
Corollary 3.1). Forde [14] studied a class of continuous, uncorrelated stochastic volatility models and computed explicitly the first two leading terms corresponding to the orders $T^{1 / 2}$ and $T^{3 / 2}$, while Forde et al. [16] obtained the same two coefficients for the Heston model with correlation. Forde and Figueroa-López [13] determined the leading-order term for the CGMY model. More generally, Tankov [27] obtained several results similar to ours in the setting of exponential Lévy models; detailed references are given below. Finally, for (arithmetic) Lévy processes $S$, the at-the-money option price is related to the power variation of order 1: if $T=\delta$ is the mesh size, $\mathrm{E}\left[\left|S_{T}\right|\right]=\mathrm{E}\left[n^{-1} \sum_{1 \leq k \leq n}\left|S_{k \delta}-S_{(k-1) \delta}\right|\right]$ for any $n \in \mathbb{N}$ by the independent and identically distributed (i.i.d.) property of the increments. Hence, it is not surprising that we will benefit from the results of Jacod [19] on the asymptotic properties of power variations.

The present paper is organized as follows. Section 2 contains the approximation result, which is stated for general martingales. Section 3 contains the analysis for the order $\sqrt{T}$ and Section 4 contains the analysis for the higher leading orders corresponding to the pure-jump case. Appendix A contains some standard results about Lévy processes that are used in the body of the text, often without further mention. We refer to the monograph of Jacod and Shiryaev [20] for any unexplained notion or notation from stochastic calculus.

\section{Approximation of the process $S$}

In this section we compare two martingales $S$ and $S^{\prime}$ with different coefficients, and study the distance $\left|S_{T}-S_{T}^{\prime}\right|$ in mean as $T \downarrow 0$. The main application to be used in the sequel is the case where $S^{\prime}$ is the Lévy approximation (1.3) of $S$. In that case, the assumption in the following result becomes a Hölder-type condition in mean for the coefficients $\sigma_{t}$ and $\kappa_{t}$ of $S$.

Proposition 2.1. Let $S$ be a martingale of the form (1.2), and let $S^{\prime}$ be a martingale of the analogous form $S^{\prime}=S_{0}^{\prime}+\sigma^{\prime} \cdot W+\kappa^{\prime}(x) *(N-F(\mathrm{~d} x) \mathrm{d} t)$ with $S_{0}^{\prime}=S_{0}$. Let $\gamma \geq 0$.

(i) If $\mathrm{E}\left[\left(\sigma_{t}-\sigma_{t}^{\prime}\right)^{2}\right]=O\left(t^{\gamma}\right)$ and $\mathrm{E}\left[\int_{\mathbb{R}}\left|\kappa_{t}(x)-\kappa_{t}^{\prime}(x)\right|^{2} F(\mathrm{~d} x)\right]=O\left(t^{\gamma}\right)$, then we have $\mathrm{E}\left[\left|S_{T}-S_{T}^{\prime}\right|^{2}\right]=O\left(T^{(1+\gamma)}\right)$ and $\mathrm{E}\left[\left|S_{T}-S_{T}^{\prime}\right|\right]=O\left(T^{(1+\gamma) / 2}\right)$.

(ii) Let $\beta \in[1,2]$. If $\mathrm{E}\left[\int_{\mathbb{R}}\left|\kappa_{t}(x)-\kappa_{t}^{\prime}(x)\right|^{\beta} F(\mathrm{~d} x)\right]=O\left(t^{\gamma}\right)$ and $\sigma \equiv 0$, then we have $\mathrm{E}\left[\left|S_{T}-S_{T}^{\prime}\right|^{\beta}\right]=O\left(T^{(1+\gamma)}\right)$ and $\mathrm{E}\left[\left|S_{T}-S_{T}^{\prime}\right|\right]=O\left(T^{(1+\gamma) / \beta}\right)$.

The assertions remain valid if $O(\cdot)$ is replaced by $o(\cdot)$ throughout.

Proof. We set $X:=S-S^{\prime}$, and denote by $X=X^{\mathrm{c}}+X^{\mathrm{d}}$ the decomposition into the continuous and purely discontinuous martingale parts; note that

$$
X^{\mathrm{c}}=\left(\sigma-\sigma^{\prime}\right) \cdot W \quad \text { and } \quad X^{\mathrm{d}}=\left(\kappa(x)-\kappa^{\prime}(x)\right) *(N-F(\mathrm{~d} x) \mathrm{d} t) .
$$

Fix $\beta \in[1,2]$ and $\gamma \geq 0$. We use the Burkholder-Davis-Gundy inequality (see, e.g. [23, Theorem IV.48]) to obtain

$$
\begin{aligned}
\mathrm{E}\left[\left|X_{T}\right|^{\beta}\right] & \leq 2^{\beta-1}\left(\mathrm{E}\left[\left|X_{T}^{\mathrm{c}}\right|^{\beta}\right]+\mathrm{E}\left[\left|X_{T}^{\mathrm{d}}\right|^{\beta}\right]\right) \\
& \leq C_{\beta}\left(\mathrm{E}\left[\left\langle X^{\mathrm{c}}, X^{\mathrm{c}}\right\rangle_{T}^{\beta / 2}\right]+\mathrm{E}\left[\left[X^{\mathrm{d}}, X^{\mathrm{d}}\right]_{T}^{\beta / 2}\right]\right)
\end{aligned}
$$

for a universal constant $C_{\beta}$ depending only on $\beta$. We first treat the pure-jump case (ii); then $X^{\mathrm{c}} \equiv 0$ and we only need to estimate the second expectation in (2.1). Recall that, for a real 
sequence $y=\left(y_{n}\right)$, the norms $\|y\|_{\ell^{p}}=\left(\sum_{n}\left|y_{n}\right|^{p}\right)^{1 / p}$ satisfy $\|y\|_{\ell^{p}} \geq\|y\|_{\ell^{q}}$ for $1 \leq p \leq$ $q<\infty$. We apply this for $p=\beta$ and $q=2$ to obtain

$$
\left[X^{\mathrm{d}}, X^{\mathrm{d}}\right]_{T}^{\beta / 2}=\left(\sum_{t \leq T}\left|\Delta X_{t}\right|^{2}\right)^{\beta / 2} \leq \sum_{t \leq T}|\Delta X|^{\beta}=\left|\kappa(x)-\kappa^{\prime}(x)\right|^{\beta} * N_{T} ;
$$

hence, using the definition of the compensator and Fubini's theorem, we have

$$
\begin{aligned}
\mathrm{E}\left[\left[X^{\mathrm{d}}, X^{\mathrm{d}}\right]_{T}^{\beta / 2}\right] & \leq \mathrm{E}\left[\left|\kappa(x)-\kappa^{\prime}(x)\right|^{\beta} * N_{T}\right] \\
& =\mathrm{E}\left[\left|\kappa(x)-\kappa^{\prime}(x)\right|^{\beta} *(F(\mathrm{~d} x) \mathrm{d} t)_{T}\right] \\
& =\int_{0}^{T} \mathrm{E}\left[\int_{\mathbb{R}}\left|\kappa_{t}(x)-\kappa_{t}^{\prime}(x)\right|^{\beta} F(\mathrm{~d} x)\right] \mathrm{d} t .
\end{aligned}
$$

By assumption, the integrand is of order $O\left(t^{\gamma}\right)$; hence, the integral is of order $O\left(T^{(1+\gamma)}\right)$ and the first assertion of (ii) follows by (2.1). The second assertion then follows by Jensen's inequality.

We now turn to case (i). Of course, the previous estimates hold, in particular, for $\beta=2$, so it remains to consider the continuous part in (2.1). For $\beta=2$, the continuous part is

$$
\mathrm{E}\left[\left\langle X^{\mathrm{c}}, X^{\mathrm{c}}\right\rangle_{T}\right]=\mathrm{E}\left[\int_{0}^{T}\left(\sigma_{t}-\sigma_{t}^{\prime}\right)^{2} \mathrm{~d} t\right]=\int_{0}^{T} \mathrm{E}\left[\left(\sigma_{t}-\sigma_{t}^{\prime}\right)^{2}\right] \mathrm{d} t,
$$

and so the conclusion is obtained as before. Finally, we note that the proof remains valid if $O(\cdot)$ is replaced by $o(\cdot)$ throughout.

We illustrate the use of Proposition 2.1 by two applications to the approximation of stochastic differential equations (SDEs). For the sake of clarity, we do not strive for minimal conditions.

Corollary 2.1. Let $f: \mathbb{R} \rightarrow \mathbb{R}$ be continuously differentiable with bounded derivative, and let $L$ be a square-integrable Lévy martingale. Then the SDE

$$
\mathrm{d} S_{t}=f\left(S_{t-}\right) \mathrm{d} L_{t}, \quad S_{0} \in \mathbb{R},
$$

has a unique solution $S$ and the Lévy process $Z_{t}=S_{0}+f\left(S_{0}\right) L_{t}$ satisfies $\mathrm{E}\left[\left|S_{T}-Z_{T}\right|\right]=O(T)$ as $T \downarrow 0$.

Proof. We recall from [23, Theorem V.67] that the SDE has a unique strong solution $S$ and that $t \mapsto \mathrm{E}\left[S_{t}^{2}\right]$ is locally bounded. The Lévy process $L$ has a representation of the form $L=c W+x *(N-F(\mathrm{~d} x) \mathrm{d} t)$ and then

$$
\begin{aligned}
& S=S_{0}+c f\left(S_{-}\right) \cdot W+\left(f\left(S_{-}\right) x\right) *(N-F(\mathrm{~d} x) \mathrm{d} t), \\
& Z=S_{0}+c f\left(S_{0}\right) \cdot W+\left(f\left(S_{0}\right) x\right) *(N-F(\mathrm{~d} x) \mathrm{d} t) .
\end{aligned}
$$

It suffices to verify the conditions of Proposition 2.1(i) for $\gamma=1$ and $S^{\prime}=Z$. In view of $S_{t}=S_{t-}$, P-a.s. for each $t$, we have $\mathrm{E}\left[\left(\sigma_{t}-\sigma_{t}^{\prime}\right)^{2}\right]=c^{2} \mathrm{E}\left[\left|f\left(S_{t}\right)-f\left(S_{0}\right)\right|^{2}\right]$ and

$$
\mathrm{E}\left[\int_{\mathbb{R}}\left|\kappa_{t}(x)-\kappa_{t}^{\prime}(x)\right|^{2} F(\mathrm{~d} x)\right]=\mathrm{E}\left[\left|f\left(S_{t}\right)-f\left(S_{0}\right)\right|^{2}\right] \int|x|^{2} F(\mathrm{~d} x) .
$$

The last integral is finite since $L$ is square integrable (see Lemma A.1(vi)), and so it suffices to show that $\mathrm{E}\left[\left|f\left(S_{t}\right)-f\left(S_{0}\right)\right|^{2}\right]=O(t)$. Now $f$ is Lipschitz continuous by assumption, so it remains to prove that

$$
\mathrm{E}\left[\left|S_{t}-S_{0}\right|^{2}\right]=O(t) .
$$


For this, in turn, it suffices to verify the conditions of Proposition 2.1(i) for $\gamma=0$ and $S^{\prime} \equiv S_{0}$ (and, hence, $\sigma^{\prime} \equiv 0$ and $\kappa^{\prime} \equiv 0$ ). Indeed, we have $\mathrm{E}\left[\left|\sigma_{t}\right|^{2}\right]=c^{2} \mathrm{E}\left[\left|f\left(S_{t}\right)\right|^{2}\right]=O(1)$ as the linear growth of $f$ ensures that $\mathrm{E}\left[f\left(S_{t}\right)^{2}\right]$ is again locally bounded like $\mathrm{E}\left[S_{t}^{2}\right]$, and similarly we have $\mathrm{E}\left[\int_{\mathbb{R}}\left|\kappa_{t}(x)\right|^{2} F(\mathrm{~d} x)\right]=\mathrm{E}\left[f\left(S_{t}\right)^{2}\right] \int|x|^{2} F(\mathrm{~d} x)=O(1)$ as $t \downarrow 0$.

Remark 2.1. If the square-integrable Lévy martingale $L$ does not have a Brownian component and if its Lévy measure $F$ satisfies

$$
\int_{\mathbb{R}}|x|^{\beta} F(\mathrm{~d} x)<\infty
$$

for some $\beta \in[1,2]$, then the assertion in Corollary 2.1 can be strengthened to $\mathrm{E}\left[\left|S_{T}-Z_{T}\right|\right]=$ $O\left(T^{2 / \beta}\right)$. In particular, this applies for $\beta=1$ when $L$ is of finite variation. The proof is as above, using part (ii) of Proposition 2.1 instead of part (i).

We give a second example where the coefficient of the SDE is not Lipschitz continuous, as this sometimes occurs in stochastic volatility models.

Corollary 2.2. Assume that $S$ solves the $S D E$

$$
\mathrm{d} S_{t}=S_{t} \sqrt{v_{t-}} \mathrm{d} W_{t}, \quad S_{0} \in \mathbb{R},
$$

where $v \geq 0$ is a càdlàg adapted process. If $t \mapsto \mathrm{E}\left[v_{t}^{2+\varepsilon}\right]$ and $t \mapsto \mathrm{E}\left[S_{t}^{4+\varepsilon}\right]$ are bounded in a neighborhood of 0 for some $\varepsilon>0$, then the Lévy process $Z_{t}=S_{0}+S_{0} \sqrt{v_{0}} W_{t}$ satisfies $\mathrm{E}\left[\left|S_{T}-Z_{T}\right|\right]=o(\sqrt{T})$.

In particular, this applies when $v$ is a square-root process, i.e. when $S$ is the Heston model.

Proof. In view of Proposition 2.1(i) applied with $\gamma=0$, it suffices to verify that

$$
\mathrm{E}\left[\left(S_{t} \sqrt{v_{t}}-S_{0} \sqrt{v_{0}}\right)^{2}\right]=o(1)
$$

note that, by the continuity of $W$, the SDE does not change if we replace $v_{-}$by $v$. As

$$
\mathrm{E}\left[\left(S_{t} \sqrt{v_{t}}-S_{0} \sqrt{v_{0}}\right)^{2}\right]=\mathrm{E}\left[S_{t}^{2} v_{t}-S_{0}^{2} v_{0}\right]+2 S_{0} \sqrt{v_{0}} \mathrm{E}\left[S_{0} \sqrt{v_{0}}-S_{t} \sqrt{v_{t}}\right],
$$

it suffices to check that $\mathrm{E}\left[S_{t}^{2} v_{t}\right] \rightarrow S_{0}^{2} v_{0}$ and $\mathrm{E}\left[S_{t} \sqrt{v_{t}}\right] \rightarrow S_{0} \sqrt{v_{0}}$ as $t \downarrow 0$. Since $S \sqrt{v}$ is right continuous, this readily follows by the Cauchy-Schwarz inequality and uniform integrability. That the assumptions are satisfied for the Heston model follows from, e.g. Cox et al. [9, Section 3] and the proof of Proposition 3.1 of [3].

\section{Option price of order $\sqrt{T}$}

The main idea in this section is to calculate the option price for $S$ from (1.2) via the approximation

$$
Z:=S_{0}+\sigma_{0} W+\kappa_{0}(x) *(N-F(\mathrm{~d} x) \mathrm{d} t) .
$$

We first have to ensure that this expression makes sense. Indeed, if $S$ is a martingale, it follows that

$$
\int_{\mathbb{R}}\left|\kappa_{t}(x)\right| \wedge\left|\kappa_{t}(x)\right|^{2} F(\mathrm{~d} x)<\infty \quad \mathrm{P} \otimes \mathrm{d} t \text {-almost everywhere, }
$$

but of course this may fail on the nullset $\{t=0\}$. Hence, we make the standing assumption that $Z$ is well defined and integrable; i.e. that $\kappa_{0}(x)$ is Borel measurable and satisfies

$$
\int_{\mathbb{R}}\left|\kappa_{0}(x)\right| \wedge\left|\kappa_{0}(x)\right|^{2} F(\mathrm{~d} x)<\infty .
$$

In any reasonable situation, we will be able to infer this condition from (3.2). 
We can now prove our result for the at-the-money option price of order $\sqrt{T}$. In fact, we describe the slightly more general situation of almost at-the-money strikes by considering a deterministic strike function $T \mapsto K_{T}$ such that $K_{T} \rightarrow S_{0}$ as $T \downarrow 0$. The main observation is that the coefficient of order $\sqrt{T}$ depends only on the initial value of $\sigma$ and that the jumps are irrelevant at this order. We denote by $\mathcal{N}$ the Gaussian distribution.

Theorem 3.1. Let $S$ be a martingale of the form (1.2), and assume that

$$
\lim _{t \downarrow 0} \mathrm{E}\left[\left(\sigma_{t}-\sigma_{0}\right)^{2}\right]=0 \quad \text { and } \quad \lim _{t \downarrow 0} \mathrm{E}\left[\int_{\mathbb{R}}\left|\kappa_{t}(x)-\kappa_{0}(x)\right|^{2} F(\mathrm{~d} x)\right]=0 .
$$

If $K_{T}=S_{0}+\theta \sqrt{T}+o(\sqrt{T})$ for some $\theta \in \mathbb{R}$ then

$$
\mathrm{E}\left[\left(S_{T}-K_{T}\right)^{+}\right]=\mathrm{E}\left[\mathcal{N}\left(-\theta, \sigma_{0}^{2}\right)^{+}\right] \sqrt{T}+o(\sqrt{T}) \text { as } T \downarrow 0 .
$$

In particular, for the at-time-money case $K \equiv S_{0}$, we have

$$
\mathrm{E}\left[\left(S_{T}-S_{0}\right)^{+}\right]=\frac{\left|\sigma_{0}\right|}{\sqrt{2 \pi}} \sqrt{T}+o(\sqrt{T}) \quad \text { as } T \downarrow 0 .
$$

Remark 3.1. (a) The form $K_{T}=S_{0}+\theta \sqrt{T}+o(\sqrt{T})$ chosen in Theorem 3.1 is in fact the only relevant one. Indeed, if the convergence $K_{T} \rightarrow S_{0}$ is slower than $\sim C \sqrt{T}$ then the leading-order asymptotics of $\mathrm{E}\left[\left(S_{T}-K_{T}\right)^{+}\right]$will simply be determined by $\left(S_{0}-K_{T}\right)^{+}$, and if it is faster, we find the same asymptotics as in the at-the-money case. As usual, we write $f(T) \sim g(T)$ if $f(T) / g(T) \rightarrow 1$ as $T \downarrow 0$. We can also note that the constant $\theta$ satisfies $\theta=\left(S_{0}-K_{T}\right) / \sqrt{T}+o(1)$ and can therefore be interpreted as a degree of moneyness for the option; a similar notion was previously used by Medvedev and Scaillet [22].

(b) Even for the class of continuous martingales, $\sqrt{T}$ is the highest order in which the option price depends only on the initial volatility $\sigma_{0}$. Indeed, assume that this were also the case for the order $T^{1 / 2+\varepsilon}$ and some $\varepsilon>0$. Set $\sigma_{t}=\sqrt{(1+2 \varepsilon) t^{2 \varepsilon}}$, and define the martingale $S_{t}=\int_{0}^{t} \sigma_{s} \mathrm{~d} W_{s}$. Then $S$ is Gaussian with variance $\operatorname{var}\left(S_{T}\right)=\int_{0}^{T} \sigma_{t}^{2} \mathrm{~d} t=T^{1+2 \varepsilon}$ and, hence,

$$
\mathrm{E}\left[S_{T}^{+}\right]=\frac{1}{\sqrt{2 \pi}} T^{1 / 2+\varepsilon} .
$$

This coefficient is of course different from that for $S^{\prime} \equiv 0$, which is another martingale with $\sigma_{0}=0$.

Let us formulate Theorem 3.1 once more, in the language preferred by practitioners. If $S>0$, the implied volatility $\sigma_{\text {impl }}(T) \in[0, \infty]$ of an at-the-money call option with maturity $T$ is defined as the solution of

$$
\mathrm{E}\left[\left(S_{T}-S_{0}\right)^{+}\right]=S_{0} \Phi\left(\frac{1}{2} \sigma_{\text {impl }}(T) \sqrt{T}\right)-S_{0} \Phi\left(-\frac{1}{2} \sigma_{\text {impl }}(T) \sqrt{T}\right),
$$

where $\Phi$ denotes the standard normal distribution function. This means that the option price coincides with its counterpart in a Black-Scholes model with volatility parameter $\sigma_{\text {impl }}(T)$. Note that $\sigma_{\text {impl }}(T)$ exists and is unique since $x \mapsto \Phi(x)-\Phi(-x)$ is strictly increasing and maps $[0, \infty]$ to $[0,1]$ and, moreover, $\mathrm{E}\left[\left(S_{T}-S_{0}\right)^{+}\right] \in\left[0, S_{0}\right]$. The following generalizes the result of [10] to unbounded coefficients and infinite-variation jumps. 
Corollary 3.1. Let $S>0$. Under the conditions of Theorem 3.1, we have $\sigma_{\text {impl }}(T) \rightarrow\left|\sigma_{0}\right| / S_{0}$, i.e. the implied volatility converges to the spot volatility of the continuous martingale part of the log price.

Proof. By the asymptotic properties of $\Phi$ (see, e.g. [1, Chapter 7]), we have $\Phi(x)-\Phi(-x) \sim$ $\sqrt{2 / \pi} x$ for small $x$. Since $\sigma_{\text {impl }}(T) \sqrt{T} \downarrow 0$ by Theorem 3.1, we obtain

$$
\Phi\left(\frac{1}{2} \sigma_{\text {impl }}(T) \sqrt{T}\right)-\Phi\left(-\frac{1}{2} \sigma_{\text {impl }}(T) \sqrt{T}\right) \sim \frac{\sigma_{\text {impl }}(T)}{\sqrt{2 \pi}} \sqrt{T} .
$$

As Theorem 3.1 yields $\mathrm{E}\left[\left(S_{T}-S_{0}\right)^{+}\right] / S_{0} \sim\left(\left|\sigma_{0}\right| / S_{0} / \sqrt{2 \pi}\right) \sqrt{T}$, the definition of $\sigma_{\text {impl }}(T)$ shows that $\sigma_{\text {impl }}(T) \rightarrow\left|\sigma_{0}\right| / S_{0}$.

Proof of Theorem 3.1. We may use the put-call parity to rewrite the call price in terms of an absolute moment: since $S$ is a martingale,

$$
\begin{aligned}
\mathrm{E}\left[\left|S_{T}-K_{T}\right|\right] & =\mathrm{E}\left[2\left(S_{T}-K_{T}\right)^{+}-S_{T}+K_{T}\right] \\
& =2 \mathrm{E}\left[\left(S_{T}-K_{T}\right)^{+}\right]-S_{0}+K_{T} .
\end{aligned}
$$

From this we also see that we may assume that $K_{T}=S_{0}+\theta \sqrt{T}$ (i.e. that the $o(\sqrt{T})$ part does not matter). By a translation we may also assume that $S_{0}=K_{0}=0$. (We exploit here the fact that we are working with a general class of martingales $S$, not necessarily positive.)

Step 1: continuous Lévy case. Assume first that $S$ is a continuous Lévy process, i.e. that $S_{t}=\sigma_{0} W_{t}$. Then

$$
\mathrm{E}\left[\left(S_{T}-\theta \sqrt{T}\right)^{+}\right]=\sqrt{T} \mathrm{E}\left[\left(\sigma_{0} W_{1}-\theta\right)^{+}\right]=\sqrt{T} \mathrm{E}\left[\mathcal{N}\left(-\theta, \sigma_{0}^{2}\right)^{+}\right] .
$$

Step 2: general Lévy case. Let $S$ be a Lévy process (i.e. $S=Z$ ), and denote by $S=S^{\mathrm{c}}+S^{\mathrm{d}}$ its decomposition into the continuous and purely discontinuous martingale parts. In view of

$$
\left|S_{T}^{\mathrm{c}}-K_{T}\right|-\left|S_{T}^{\mathrm{d}}\right| \leq\left|S_{T}-K_{T}\right| \leq\left|S_{T}^{\mathrm{c}}-K_{T}\right|+\left|S_{T}^{\mathrm{d}}\right|
$$

and step 1, it suffices to show that $\mathrm{E}\left[\left|S_{T}^{\mathrm{d}}\right|\right]$ is of order $o(\sqrt{T})$. To relax the notation, let us assume that $S^{\mathrm{d}}=S$. We can further decompose $S$ into a martingale with bounded jumps, which is in particular square integrable, and a compound Poisson process $X$ which is integrable due to (3.3). We can check by direct calculation or by an application of Theorem 4.1 below that $\mathrm{E}\left[\left|X_{T}\right|\right]=O(T)$. That is, we may even assume that $S$ is a square-integrable, pure-jump Lévy martingale. Then

$$
\frac{\left|S_{T}\right|}{\sqrt{T}} \rightarrow 0 \quad \text { in probability as } T \downarrow 0 ;
$$

see, e.g. [19, Lemma 4.1]. To conclude that $\mathrm{E}\left[\left|S_{T}\right|\right] / \sqrt{T} \rightarrow 0$, it suffices to show the uniform integrability of $\left\{S_{T} / \sqrt{T}\right\}_{T>0}$. But this set is even bounded in $L^{2}(P)$ as $S$ is square integrable; indeed, $\left\|S_{T} / \sqrt{T}\right\|_{L^{2}(P)}^{2}=\mathrm{E}\left[[S, S]_{1}\right]$ due to the relation $\mathrm{E}\left[S_{T}^{2}\right]=\mathrm{E}\left[[S, S]_{T}\right]=T \mathrm{E}\left[[S, S]_{1}\right]$.

Step 3: general case. When $S$ is as in the theorem, we approximate $S$ by the Lévy process $Z$ from (3.1). The assumptions of Proposition 2.1(i) are satisfied for $S^{\prime}=Z$ and the order $o(1)$; hence, we obtain that $\mathrm{E}\left[\left|S_{T}-Z_{T}\right|\right]$ is of order $o(\sqrt{T})$. In view of step 2 applied to $Z$ and

$$
\mathrm{E}\left[\left|Z_{T}-K_{T}\right|\right]-\mathrm{E}\left[\left|S_{T}-Z_{T}\right|\right] \leq \mathrm{E}\left[\left|S_{T}-K_{T}\right|\right] \leq \mathrm{E}\left[\left|Z_{T}-K_{T}\right|\right]+\mathrm{E}\left[\left|S_{T}-Z_{T}\right|\right],
$$

the proof is complete. 


\section{Option prices with higher leading orders}

In this section we consider pure-jump martingales

$$
S=S_{0}+\kappa(x) *(N-F(\mathrm{~d} x) \mathrm{d} t),
$$

i.e. we set $\sigma \equiv 0$ in (1.2). Then the term of order $\sqrt{T}$ in Theorem 3.1 vanishes and the leading order is higher than $1 / 2$. We will again use the approximation result from Section 2 to reduce to the Lévy case and consider

$$
Z=S_{0}+\kappa_{0}(x) *(N-F(\mathrm{~d} x) \mathrm{d} t) .
$$

However, this case is now more involved since the results depend on the properties of the Lévy measure. We recall the standing assumption (3.3) which ensures that $Z$ is well defined and integrable.

\subsection{Finite variation}

We first treat the case when $S$ is of finite variation, which leads to the highest possible (nontrivial) convergence rate for the at-the-money option price. Indeed, the following result shows that this class of price processes is characterized by the rate $O(T)$.

Theorem 4.1. Let $S$ be a pure-jump martingale of the form (4.1), and assume that

$$
\lim _{t \downarrow 0} \mathrm{E}\left[\int_{\mathbb{R}}\left|\kappa_{t}(x)-\kappa_{0}(x)\right| F(\mathrm{~d} x)\right]=0 .
$$

Then the following assertions are equivalent:

(i) $S$ is of finite variation on $[0, T]$ for some $T>0$,

(ii) $\mathrm{E}\left[\left(S_{T}-S_{0}\right)^{+}\right]=O(T)$ as $T \downarrow 0$.

In that case,

$$
\mathrm{E}\left[\left(S_{T}-S_{0}\right)^{+}\right]=\frac{1}{2} C T+o(T) \text { as } T \downarrow 0,
$$

where $C:=\int_{\mathbb{R}}\left|\kappa_{0}(x)\right| F(\mathrm{~d} x)+\left|\int_{\mathbb{R}} \kappa_{0}(x) F(\mathrm{~d} x)\right|$.

Proof. As in the proof of Theorem 3.1, we may assume that $S_{0}=0$ and then we have $\mathrm{E}\left[\left|S_{T}\right|\right]=2 \mathrm{E}\left[S_{T}^{+}\right]$. Let us first clarify the meaning of (i) under the given conditions. The assumed convergence implies in particular that

$$
\mathrm{E}\left[\int_{0}^{T} \int_{\mathbb{R}}\left|\kappa_{t}(x)-\kappa_{0}(x)\right| F(\mathrm{~d} x) \mathrm{d} t\right]<\infty \quad \text { for some } T>0 .
$$

Owing to this fact, the following assertions are actually equivalent to (i):

(a) $Z$ is of integrable variation,

(b) $S$ is of integrable variation on $[0, T]$ for some $T>0$.

Indeed, assume that (i) holds. Then $S=S^{1}-S^{2}$ on [0,T] for two increasing processes $S^{1}$ and $S^{2}$ with $S_{0}^{1}=S_{0}^{2}=0$. Since the jumps of the martingale $S$ are integrable, the positive stopping time $\tau:=\inf \left\{0 \leq t \leq T: S_{t}^{1}+S_{t}^{2} \geq 1\right\}$ is such that $S$ is of integrable variation on $\llbracket 0, \tau \rrbracket$. 
By [20, Theorem II.1.33b], this implies that $\mathrm{E}\left[\int_{0}^{\tau} \int_{\mathbb{R}}\left|\kappa_{t}(x)\right| F(\mathrm{~d} x) \mathrm{d} t\right]<\infty$. Now we can use the fact that the product $\tau \int_{\mathbb{R}}\left|\kappa_{0}(x)\right| F(\mathrm{~d} x)=\int_{0}^{\tau} \int_{\mathbb{R}}\left|\kappa_{0}(x)\right| F(\mathrm{~d} x) \mathrm{d} t$ is bounded by

$$
\int_{0}^{\tau} \int_{\mathbb{R}}\left|\kappa_{t}(x)-\kappa_{0}(x)\right| F(\mathrm{~d} x) \mathrm{d} t+\int_{0}^{\tau} \int_{\mathbb{R}}\left|\kappa_{t}(x)\right| F(\mathrm{~d} x) \mathrm{d} t<\infty \quad \text { P-a.s. }
$$

to conclude via Lemma A.1(vii) that $Z$ is of integrable variation. Furthermore, (a) together with (4.3) yields $\mathrm{E}\left[\int_{0}^{T} \int_{\mathbb{R}}\left|\kappa_{t}(x)\right| F(\mathrm{~d} x) \mathrm{d} t\right]<\infty$, which is (b) by [20, Theorem II.1.33b], and, clearly, (b) implies (i).

Step 1: Lévy case. We first assume that $S=Z$. Moreover, we replace (i) by the equivalent condition (a).

(ii) implies (a). We consider an increasing sequence of continuous functions $f_{n}$ on $\mathbb{R}$ satisfying

$$
0 \leq f_{n}(x) \leq|x| \wedge n, \quad f_{n}(x)=0 \quad \text { for }|x|<\frac{1}{n}, \quad \lim _{n} f_{n}(x)=|x|
$$

for all $x \in \mathbb{R}$ and $n \geq 1$. For each $n$, we have

$$
\liminf _{T \downarrow 0} \frac{1}{T} \mathrm{E}\left[\left|Z_{T}\right|\right] \geq \liminf _{T \downarrow 0} \frac{1}{T} \mathrm{E}\left[f_{n}\left(Z_{T}\right)\right]=\int_{\mathbb{R}} f_{n}\left(\kappa_{0}(x)\right) F(\mathrm{~d} x),
$$

where the equality follows from Sato [26, Corollary 8.9] since $f_{n}$ vanishes in a neighborhood of the origin (this holds for any Lévy measure). By monotone convergence as $n \rightarrow \infty$ we obtain

$$
\liminf _{T \downarrow 0} \frac{1}{T} \mathrm{E}\left[\left|Z_{T}\right|\right] \geq \int\left|\kappa_{0}(x)\right| F(\mathrm{~d} x) .
$$

The left-hand side is finite by assumption; hence, $Z$ is of integrable variation.

(a) implies (ii). If $Z$ is of integrable variation, its total variation process $\operatorname{var}(Z)_{t}:=\int_{0}^{t}\left|\mathrm{~d} Z_{s}\right|$ is an integrable Lévy subordinator and, by Lemma A.1(vii),

$$
\frac{1}{T} \mathrm{E}\left[\operatorname{var}(Z)_{T}\right]=\mathrm{E}\left[\operatorname{var}(Z)_{1}\right]=\int\left|\kappa_{0}(x)\right| F(\mathrm{~d} x)+\left|\int \kappa_{0}(x) F(\mathrm{~d} x)\right|=C .
$$

Since $\left|Z_{T}\right| \leq \operatorname{var}(Z)_{T}$, we conclude that

$$
\underset{T \downarrow 0}{\limsup } \frac{1}{T} \mathrm{E}\left[\left|Z_{T}\right|\right] \leq C .
$$

For the converse inequality, we consider the function $g_{n}(x)=|x| \wedge n$ for $n \geq 1$. Since $Z$ is of finite variation, we obtain, for each $n$,

$$
\liminf _{T \downarrow 0} \frac{1}{T} \mathrm{E}\left[\left|Z_{T}\right|\right] \geq \lim _{T \downarrow 0} \frac{1}{T} \mathrm{E}\left[g_{n}\left(Z_{T}\right)\right]=\int g_{n}\left(\kappa_{0}(x)\right) F(\mathrm{~d} x)+\left|\int \kappa_{0}(x) F(\mathrm{~d} x)\right|
$$

as a consequence of [19, Theorem 2.1(i)(c)] since the increments of $Z$ are i.i.d. (see also [19, Equation (5.8)]). Applying monotone convergence as $n \rightarrow \infty$ to the right-hand side, we conclude that

$$
\liminf _{T \downarrow 0} \frac{1}{T} \mathrm{E}\left[\left|Z_{T}\right|\right] \geq C .
$$

Hence, we have proved the claimed convergence rate for the case $S=Z$. 
Step 2: general case. Under the stated assumption, Proposition 2.1(ii) with $\beta=1$ and $\gamma=0$ yields $\mathrm{E}\left[\left|S_{T}-Z_{T}\right|\right]=o(T)$ as $T \downarrow 0$. Hence, (ii) holds for $S$ if and only if it holds for $Z$ and so step 1 yields the equivalence of (i) and (ii). Moreover, the leading constant $C$ is the same as for $Z$ since the approximation error is of order $o(T)$.

Remark 4.1. (a) For exponential Lévy processes, where $\kappa(x)=\mathrm{e}^{x}-1$, the formula in Theorem 4.1(ii) was previously obtained by Tankov [27]. We thank the referee for pointing out this reference.

(b) The constant $C$ has the following feature. For a given absolute moment $\mu:=\int\left|\kappa_{0}(x)\right| F(\mathrm{~d} x)$ of the Lévy measure of $Z$, the value of $C$ may range from $\mu$ to $2 \mu$ depending on $\int \kappa_{0}(x) F(\mathrm{~d} x)$. In particular, $C$ is minimal if the jumps are symmetric, and maximal if all jumps have the same sign.

(c) As in Corollary 3.1, it follows from Theorem 4.1 that the implied volatility satisfies $\sigma_{\text {impl }}(T) \sim \sqrt{\pi / 2} C / S_{0} \sqrt{T}$ as $T \downarrow 0$.

(d) The following observation from step 1 in the proof of Theorem 4.1 seems worth recording: if $Z$ is a Lévy martingale of finite variation, then the three functions $T \mapsto \mathrm{E}\left[\left|Z_{T}\right|\right]$, $T \mapsto \mathrm{E}\left[\sup _{t \leq T}\left|Z_{t}\right|\right]$, and $T \mapsto \mathrm{E}\left[\operatorname{var}(Z)_{T}\right]$ all converge to 0 as $T \downarrow 0$ with the same leading term $C T$.

\subsection{Infinite variation}

We now turn to pure-jump processes with infinite variation. By the previous results we know that the leading order for the at-the-money option price has to be strictly between $\sqrt{T}$ and $T$; however, it need not be a power of $T$. The class of possible Lévy measures at time 0 is very rich and it is unclear how to compute the exact order in general. A look at existing financial models suggests imposing an additional structure which will pin down an order of parametric form. The base case is the $\alpha$-stable Lévy measure which is given by

$$
v(\mathrm{~d} x)=\frac{g(x)}{|x|^{1+\alpha}} \mathrm{d} x, \quad g(x):=\beta_{-} \mathbf{1}_{(-\infty, 0)}(x)+\beta_{+} \mathbf{1}_{(0, \infty)}(x)
$$

for $\alpha \in(0,2)$ and two nonnegative constants $\beta_{+}$and $\beta_{-}$. More precisely, $v$ corresponds to a Lévy process $L_{t}$ following a stable law with index of stability $\alpha$, skewness parameter $\beta=\left(\beta_{+}-\beta_{-}\right) /\left(\beta_{+}+\beta_{-}\right)$, shift parameter $\mu=0$, and scale parameter $c=\left(\beta_{+}+\beta_{-}\right)^{1 / \alpha} t^{1 / \alpha}$. In the nondegenerate case, $\beta_{+}+\beta_{-}>0$, the process $L$ has infinite variation if and only if $\alpha \in[1,2)$. For $\alpha \in(1,2)$, the first moment $\mathrm{E}\left[\left|L_{t}\right|\right]$ exists whereas, for $\alpha \in(0,1]$, this is not the case and in particular $L$ cannot be a martingale. On the other hand, only the small jumps are relevant for the option price asymptotics of order smaller than $O(T)$, which leads us to the following definition.

Definition 4.1. Let $\alpha_{+}, \alpha_{-} \in(0,2)$. A Lévy process is said to have $\left(\alpha_{+}, \alpha_{-}\right)$-stable-like small jumps if its Lévy measure $v$ is of the form

$$
v(\mathrm{~d} x)=\left(\frac{f(x)}{|x|^{1+\alpha_{-}}} \mathbf{1}_{(-\infty, 0)}(x)+\frac{f(x)}{|x|^{1+\alpha_{+}}} \mathbf{1}_{(0, \infty)}(x)\right) \mathrm{d} x
$$

for a Borel function $f \geq 0$ whose left and right limits at 0 ,

$$
f_{+}:=\lim _{x \downarrow 0} f(x) \quad \text { and } \quad f_{-}:=\lim _{x \uparrow 0} f(x),
$$

exist and satisfy $f(x)-f_{+}=O(x)$ as $x \downarrow 0$ and $f(x)-f_{-}=O(x)$ as $x \uparrow 0$. 
This class includes most of the processes used in financial modeling, e.g. the tempered stable and in particular the CGMY processes, of which the variance gamma process is a special case, or the normal inverse Gaussian process. We refer the reader to [8, Section 4.5] for more information on these models. We observe that if the driving Lévy process of an SDE as in Corollary 2.1 is chosen from this class, then the process $Z$ defined in the same corollary is again of the same type; merely the constants $f_{+}$and $f_{-}$change.

Since $\alpha_{+} \vee \alpha_{-} \in(0,1)$ implies that the jumps are of finite variation (cf. Theorem 4.1), we are interested here only in the case $\alpha_{+} \vee \alpha_{-} \in[1,2)$. In that case we will see that the larger of the values $\alpha_{+}$and $\alpha_{-}$determines the leading order.

The statement of our main result requires the following constants. For $f_{+}, f_{-} \geq 0$ and $\alpha \in(1,2)$, we set $C(\alpha, 0,0):=0$ and if $f_{+}+f_{-}>0$ then

$$
\begin{aligned}
C\left(\alpha, f_{+}, f_{-}\right):= & \frac{2}{\pi}\left(f_{+}+f_{-}\right)^{1 / \alpha} \Gamma\left(1-\frac{1}{\alpha}\right)\left[1+\left(\frac{f_{+}-f_{-}}{f_{+}+f_{-}}\right)^{2} \tan ^{2}\left(\frac{\alpha \pi}{2}\right)\right]^{1 / 2 \alpha} \\
& \times \cos \left(\frac{1}{\alpha} \arctan \left(\frac{f_{+}-f_{-}}{f_{+}+f_{-}} \tan \left(\frac{\alpha \pi}{2}\right)\right)\right)
\end{aligned}
$$

where $\Gamma$ denotes the usual gamma function. For $\alpha_{+}, \alpha_{-} \in(0,2)$ such that $\alpha_{+} \vee \alpha_{-} \in(1,2)$, we then define

$$
C\left(\alpha_{+}, \alpha_{-}, f_{+}, f_{-}\right):= \begin{cases}C\left(\alpha_{+}, f_{+}, f_{-}\right), & \alpha_{+}=\alpha_{-}, \\ C\left(\alpha_{+}, f_{+}, 0\right), & \alpha_{+}>\alpha_{-}, \\ C\left(\alpha_{-}, 0, f_{-}\right), & \alpha_{+}<\alpha_{-} .\end{cases}
$$

Theorem 4.2. Let $S$ be a pure-jump martingale of the form (4.1) such that the Lévy process $Z$ from (4.2) has $\left(\alpha_{+}, \alpha_{-}\right)$-stable-like small jumps, and let $\alpha:=\alpha_{+} \vee \alpha_{-}$. Assume that there exist $\beta \in[1,2]$ and $\gamma \geq 0$ such that

$$
\mathrm{E}\left[\int_{\mathbb{R}}\left|\kappa_{t}(x)-\kappa_{0}(x)\right|^{\beta} F(\mathrm{~d} x)\right]=o\left(t^{\gamma}\right) \text { and } \frac{\beta}{1+\gamma} \leq \alpha .
$$

(i) If $\alpha \in(1,2)$ then

$$
\mathrm{E}\left[\left(S_{T}-S_{0}\right)^{+}\right]=\frac{1}{2} C\left(\alpha_{+}, \alpha_{-}, f_{+}, f_{-}\right) T^{1 / \alpha}+o\left(T^{1 / \alpha}\right) \quad \text { as } T \downarrow 0 .
$$

(ii) If $\alpha_{+}=\alpha_{-}=1$ and $f_{+}=f_{-}$, then

$$
\mathrm{E}\left[\left(S_{T}-S_{0}\right)^{+}\right]=\frac{1}{2}\left(f_{+}+f_{-}\right) T|\log T|+o(T|\log T|) \quad \text { as } T \downarrow 0 .
$$

Remark 4.2. (a) For exponential Lévy processes, a result similar to Theorem 4.2(i) is obtained in [27]. There, the condition on the jumps in formulated in a slightly different way and, hence, our theorem is not a strict generalization. More specifically, in [27], the author dealt with Lévy processes whose characteristic function resembles that of a stable process. On the other hand, we consider processes whose jump measures resemble the jump measure of a stable process around the origin, which seems more convenient beyond Lévy models. For a stable process, one readily verifies that the two expressions for the small-time limit indeed coincide, once the relationship between the characteristic function and the jump measure (cf., e.g. [25, Chapter I.1]) is taken into account.

The special case of a CGMY model is also treated in [13]. 
(b) In Theorem 4.2(i), the continuity assumption on $\kappa_{t}(x)$ is satisfied in particular if

$$
\mathrm{E}\left[\int_{\mathbb{R}}\left|\kappa_{t}(x)-\kappa_{0}(x)\right|^{2} F(\mathrm{~d} x)\right]=O(t) .
$$

For example, this holds in the setting of the Lévy-driven SDE of Corollary 2.1 (see the proof of that result).

(c) In the limit $\alpha \uparrow 2$, which corresponds to the Brownian case, we obtain the order $\sqrt{T}$ as in Theorem 3.1. On the other hand, the limit $\alpha \downarrow 1$ in Theorem 4.2(i) does not yield the order obtained in Theorem 4.2(ii) and the leading constants explode since $\lim _{a \downarrow 0} \Gamma(a)=+\infty$.

(d) Theorem 4.2 still holds if the regularity of $f$ in Definition 4.1 is weakened as follows. Instead of $f(x)-f_{ \pm}=O(|x|)$, it is sufficient to have $f(x)-f_{ \pm}=O\left(|x|^{\varrho}\right)$ for some $\varrho>\alpha / 2$. The proof is identical.

(e) As in Corollary 3.1, we can deduce that the implied volatility satisfies

$$
\sigma_{\text {impl }}(T) \sim \frac{\sqrt{\pi / 2} C\left(\alpha_{+}, \alpha_{-}, f_{+}, f_{-}\right)}{S_{0} T^{1 / \alpha-1 / 2}}
$$

in the setting of Theorem 4.2(i) and $\sigma_{\text {impl }}(T) \sim \sqrt{\pi / 2}\left(f_{+}+f_{-}\right) / S_{0} \sqrt{T}|\log T|$ in the setting of Theorem 4.2(ii).

(f) The following consequence of a result due to Luschgy and Pagès (see [21, Theorem 3]) complements Theorem 4.2(ii). If $S$ is a $(1,1)$-stable-like Lévy process, possibly with $f_{+} \neq f_{-}$, then $\mathrm{E}\left[\left(S_{T}-S_{0}\right)^{+}\right]=O(T|\log T|)$ still holds. However, their method only yields an upper bound and not that the leading order is indeed $\sim C T|\log T|$. As in the proof below, we can infer that the same bound holds if $S$ is not a Lévy process but satisfies the assumptions of Theorem 4.2(ii), excluding $f_{+}=f_{-}$.

4.2.1. Proof of Theorem 4.2. The plan for the proof of Theorem 4.2 is as follows. We will again reduce the general martingale to the Lévy case using Proposition 2.1. Since the first absolute moment is known for stable processes, the main step for part (i) will be to estimate the error made when replacing a stable-like Lévy process by a true stable process with index $\alpha=\alpha_{+} \vee \alpha_{-}>1$. For part (ii), the situation is slightly different as the 1 -stable process fails to have a first moment; in this case we will instead use the normal inverse Gaussian as a reference process.

In a first step we show more generally that we can pass with an error of order $O(T)$ from one Lévy process to another when the small jumps have a similar behavior.

Lemma 4.1. Let $L$ and $L^{\prime}$ be pure-jump Lévy martingales with Lévy measures $v$ and $v^{\prime}$, respectively. Suppose that, for some $\delta>0$, the Radon-Nikodym derivative

$$
\psi(x)=\frac{\mathrm{d}\left(\left.v^{\prime}(\mathrm{d} x)\right|_{[-\delta, \delta]}\right)}{\mathrm{d}\left(\left.v(\mathrm{~d} x)\right|_{[-\delta, \delta]}\right)}
$$

of the measures restricted to $[-\delta, \delta]$ exists and that $\int_{-\delta}^{\delta}|\psi(x)-1|^{2} v(\mathrm{~d} x)<\infty$. Then $\mathrm{E}\left[\left|L_{T}\right|\right]=$ $\mathrm{E}\left[\left|L_{T}^{\prime}\right|\right]+O(T)$ as $T \downarrow 0$.

Proof. Let $L=x *(\mu-v(\mathrm{~d} x) \mathrm{d} t)$ be the canonical representation of $L$; here $\mu$ is the random measure associated with the jumps of $L$. We decompose $L$ into $L=L^{\leq \delta}+L^{>\delta}$, where the Lévy process

$$
L^{\leq \delta}:=x \mathbf{1}_{\{|x| \leq \delta\}} *(\mu-v(\mathrm{~d} x) \mathrm{d} t)
$$


is obtained by truncating the jumps at magnitude $\delta$. Then $L^{>\delta}=L-L^{\leq \delta}$ is of finite variation and Theorem 4.1 yields

$$
\mathrm{E}\left[\left|L_{T}^{>\delta}\right|\right]=O(T) \quad \text { as } T \downarrow 0 .
$$

Hence, we may assume that $L=L^{\leq \delta}$, i.e. that the jumps are bounded by $\delta$ in absolute value, or, equivalently, that $v$ is concentrated on $[-\delta, \delta]$. The integrability assumption on $\psi$ ensures that $Y:=(\psi(x)-1) *(\mu-v(\mathrm{~d} x) \mathrm{d} t)$ is a square-integrable Lévy martingale; moreover, $\psi \geq 0$ implies that $\Delta Y \geq-1$. Hence, the stochastic exponential

$$
D:=\mathscr{E}(Y)=\mathscr{E}((\psi(x)-1) *(\mu-v(\mathrm{~d} x) \mathrm{d} t))
$$

is a nonnegative square-integrable martingale (cf. Lemma A.1(x)). We define the probability measure $\mathrm{Q} \ll \mathrm{P}$ on $\mathcal{F}_{1}$ by $\mathrm{dQ} / \mathrm{dP}=D_{1}$. Then it follows from the Girsanov-Jacod-Mémin theorem (cf. [20, Theorem III.3.24]) that, under Q, the process $\left(L_{t}\right)_{0 \leq t \leq 1}$ is Lévy with triplet $\left(b_{\mathrm{Q}}, 0, v_{\mathrm{Q}}\right)$ relative to the truncation function $h(x)=x$, where $v_{\mathrm{Q}}(\mathrm{d} x)=\psi(x) v(\mathrm{~d} x)=$ $\mathbf{1}_{[-\delta, \delta]} v^{\prime}(\mathrm{d} x)$ and

$$
b_{\mathrm{Q}}=\int_{-\delta}^{\delta} x(\psi(x)-1) v(\mathrm{~d} x)
$$

this integral is finite due to the assumption on $\psi$ and Hölder's inequality. After subtracting the linear drift, which is of order $O(T)$, the Q-distribution of $L$ therefore coincides with the P-distribution of $L^{\prime \leq \delta}$, which is obtained from $L^{\prime}$ by truncating the jumps. As before, we may assume that $L^{\prime}=L^{\prime \leq \delta}$. To summarize, we have

$$
\mathrm{E}_{\mathrm{Q}}\left[\left|L_{T}\right|\right]=\mathrm{E}\left[\left|L_{T}^{\prime}\right|\right]+O(T),
$$

and, hence, it suffices to show that $\mathrm{E}_{\mathrm{Q}}\left[\left|L_{T}\right|\right]-\mathrm{E}\left[\left|L_{T}\right|\right]=O(T)$. Indeed, $\mathrm{E}_{\mathrm{Q}}\left[\left|L_{T}\right|\right]=$ $\mathrm{E}\left[D_{T}\left|L_{T}\right|\right]$, Hölder's inequality, and Lemma A.1(vi) and (x) yield

$$
\begin{aligned}
\left|\mathrm{E}_{Q}\left[\left|L_{T}\right|\right]-\mathrm{E}\left[\left|L_{T}\right|\right]\right| & \leq \mathrm{E}\left[\left|D_{T}-1\right|\left|L_{T}\right|\right] \\
& \leq\left\{\mathrm{E}\left[\left(D_{T}-1\right)^{2}\right] \mathrm{E}\left[L_{T}^{2}\right]\right\}^{1 / 2} \\
& =\left\{\left(\mathrm{E}\left[D_{T}^{2}\right]-1\right) \mathrm{E}\left[L_{T}^{2}\right]\right\}^{1 / 2} \\
& =\left\{\left(\mathrm{e}^{T\langle Y, Y\rangle_{1}}-1\right) T\langle L, L\rangle_{1}\right\}^{1 / 2} \\
& =O(T),
\end{aligned}
$$

where we have used the fact that both $Y$ and $L=L^{\leq \delta}$ are square integrable.

Proof of Theorem 4.2(i). As in the proof of Theorem 3.1, we may assume that $S_{0}=0$; then we have $\mathrm{E}\left[\left|S_{T}\right|\right]=2 \mathrm{E}\left[S_{T}^{+}\right]$.

Step 1: $\alpha$-stable Lévy case. We first note the result for $Z$, and under the additional assumptions that $f(x)=f_{-} \mathbf{1}_{(-\infty, 0)}(x)+f_{+} \mathbf{1}_{(0, \infty)}(x)$ and $\alpha_{+}=\alpha_{-}=: \alpha$. Then $Z$ is a centered $\alpha$-stable Lévy motion with $\alpha \in(1,2)$, and in this case it is known that

$$
\mathrm{E}\left[\left|Z_{T}\right|\right]=C\left(\alpha, f_{+}, f_{-}\right) T^{1 / \alpha}
$$

see [25, Property 1.2.17].

Step 2: stable-like Lévy case with $\alpha_{+}=\alpha_{-}$. We again consider $Z$ for the special case $\alpha_{+}=\alpha_{-}=: \alpha$, but now we let $f$ be an arbitrary function satisfying $f(x)-f_{+}=O(x)$ as $x \uparrow 0$ and $f(x)-f_{-}=O(x)$ as $x \downarrow 0$. For $\delta>0$, we define the function

$$
\psi(x):=\mathbf{1}_{[-\delta, 0]}(x)\left(\frac{f_{-}}{f(x)}+\mathbf{1}_{\left\{f_{-}=0\right\}}\right)+\mathbf{1}_{(0, \delta]}(x)\left(\frac{f_{+}}{f(x)}+\mathbf{1}_{\left\{f_{+}=0\right\}}\right),
$$


where we use the convention that $0 / 0:=0$. We have $|\psi(x)-1|=O(|x|)$. Indeed, consider the case $f_{+}>0$. Then, for small enough $x>0$, we have $f(x) \geq f_{+} / 2>0$ and, thus,

$$
|\psi(x)-1|=\left|\frac{f_{+}-f(x)}{f(x)}\right| \leq \frac{2}{f_{+}}\left|f_{+}-f(x)\right|=O(x) \quad \text { as } x \downarrow 0 .
$$

The case $f_{+}=0$ is trivial and $x<0$ is treated in the same way. As a result, by choosing small enough $\delta$ we can find a constant $M>0$ such that

$$
|\psi(x)-1| \leq M|x|, \quad x \in[-\delta, \delta] .
$$

In particular, $\psi$ satisfies the integrability assumption of Lemma 4.1. In the sequel, we denote by $v$ the Lévy measure of $Z$, i.e. $v(\cdot)=F\left(\kappa_{0}^{-1}(\cdot)\right)$.

Step $2 a: f_{+}>0$ and $f_{-}>0$. In this case we have

$$
v^{\prime}(\mathrm{d} x):=\psi(x) \nu(\mathrm{d} x)=\left(\mathbf{1}_{[-\delta, 0)}(x) \frac{f_{-}}{|x|^{1+\alpha}}+\mathbf{1}_{(0, \delta]} \frac{f_{+}}{|x|^{1+\alpha}}(x)\right) \mathrm{d} x .
$$

Note that $v^{\prime}$ is the Lévy measure of an $\alpha$-stable Lévy motion whose jumps were truncated at magnitude $\delta$. As above, this truncation changes the option price only at the order $O(T)$. Now Lemma 4.1 and step 1 yield

$$
\mathrm{E}\left[\left|Z_{T}\right|\right]=C\left(\alpha, f_{+}, f_{-}\right) T^{1 / \alpha}+O(T) .
$$

Step $2 b: f_{+}=0$ and $f_{-}>0$. Note that in this case the formula for $v^{\prime}$ is not the desired one in general, since we have $f(x)$ instead of $f_{+}$in

$$
v^{\prime}(\mathrm{d} x)=\psi(x) \nu(\mathrm{d} x)=\left(\mathbf{1}_{[-\delta, 0)}(x) \frac{f_{-}}{|x|^{1+\alpha}}+\mathbf{1}_{(0, \delta]} \frac{f(x)}{|x|^{1+\alpha}}(x)\right) \mathrm{d} x .
$$

However, the previous argument does apply if $f(x)=f_{+}=0$ for all $x>0$, and we will reduce to this case. Indeed, Lemma 4.2 stated below shows that the positive jumps of $Z$ are of finite variation and, hence, of integrable variation by (3.3). By subtracting these jumps from $Z$ and compensating, we achieve $f(x)=f_{+}=0$ for all $x>0$ and Theorem 4.1 shows that this manipulation affects the option price only at the order $O(T)$. Hence, we may conclude as in step 2a to obtain (4.4).

The case where $f_{+}>0$ and $f_{-}=0$ is analogous. Finally, if $f_{+}$and $f_{-}$both vanish, Lemma 4.2 shows that $Z$ is of finite variation and Theorem 4.1 yields an option price of order $O(T)$. Hence, (4.4) again holds since $C(\alpha, 0,0)=0$.

Step 3: stable-like Lévy case with $\alpha_{+} \neq \alpha_{-}$. We consider the case where $\alpha:=\alpha_{+}>\alpha_{-}$. The idea is to reduce to the case where $\alpha_{+}=\alpha_{-}$but $f_{-}=0$, i.e. we get rid of all the negative jumps and show that this induces an error of order $o\left(T^{1 / \alpha}\right)$.

Let $\mu$ be the random measure associated with the jumps of $Z$. By setting

$$
Z^{+}:=x^{+} *(\mu-v(\mathrm{~d} x) \mathrm{d} t) \quad \text { and } \quad Z^{-}:=x^{-} *(\mu-v(\mathrm{~d} x) \mathrm{d} t),
$$

we decompose $Z=Z^{+}+Z^{-}$, where $Z^{+}$and $Z^{-}$are Lévy martingales having only positive and negative jumps, respectively, and Lévy measures given by $v^{+}(\mathrm{d} x)=\mathbf{1}_{(0, \infty)}(x) v(\mathrm{~d} x)$ and $v^{-}(\mathrm{d} x)=\mathbf{1}_{(-\infty, 0)}(x) v(\mathrm{~d} x)$. Note the abuse of notation: $x^{+}$and $x^{-}$refer to the positive and negative parts of $x$ while $Z^{ \pm}$and $v^{ \pm}$are new symbols. 
We observe that $Z^{+}$has $\left(\alpha_{+}, \alpha_{+}\right)$-stable-like jumps, where the corresponding function $f^{\prime}$ in Definition 4.1 satisfies $f^{\prime}(x)=0$ for $x<0$. In particular, the left limit is $f_{-}^{\prime}=0$. The martingale $Z^{-}$has analogous properties for $\alpha_{-}$.

From step 2 we know that $\mathrm{E}\left[\left|Z_{T}^{-}\right|\right]=O\left(T^{1 / \alpha_{-}}\right)$if $\alpha_{-} \in(1,2)$, and we have also seen that $\mathrm{E}\left[\left|Z_{T}^{-}\right|\right]=O(T)$ if $\alpha_{-} \in(0,1)$, due to finite variation. For the remaining case $\alpha_{-}=1$, we have $\mathrm{E}\left[\left|Z_{T}^{-}\right|\right]=O(T|\log T|)$ by Remark 4.2(f). As $\alpha_{-}<\alpha_{+}$and $\alpha_{+}>1$, we therefore have

$$
\mathrm{E}\left[\left|Z_{T}^{-}\right|\right]=o\left(T^{1 / \alpha_{+}}\right)
$$

in all three cases for $\alpha_{-}$. On the other hand, we know from step 2 applied to $\alpha_{+}$that $\mathrm{E}\left[\left|Z_{T}^{+}\right|\right] \sim$ $C T^{1 / \alpha_{+}}$. Therefore, the leading-order coefficient for $Z$ is the same as for $Z^{+}$, i.e.

$$
\mathrm{E}\left[\left|Z_{T}\right|\right]=C\left(\alpha_{+}, f_{+}, 0\right) T^{1 / \alpha_{+}}+o\left(T^{1 / \alpha_{+}}\right) .
$$

The case $\alpha_{+}<\alpha_{-}$is analogous.

Step 4: general case. Proposition 2.1(ii) implies that

$$
\mathrm{E}\left[\left|S_{T}-Z_{T}\right|\right]=o\left(T^{(1+\gamma) / \beta}\right)
$$

and, in particular, $\mathrm{E}\left[\left|S_{T}-Z_{T}\right|\right]=o\left(T^{1 / \alpha}\right)$. In view of the previous steps, the proof is complete.

The following result was used in the preceding proof.

Lemma 4.2. Let L be a Lévy process with $\left(\alpha_{+}, \alpha_{-}\right)$-stable-like small jumps for some $\alpha_{+}, \alpha_{-} \in$ $(0,2)$. If the function $f$ from Definition 4.1 satisfies $f_{+}=0$ then the positive jumps of $L$ are of finite variation, that is, $\sum_{t \leq T}\left(\Delta L_{t}\right)^{+}<\infty$ for any $T<\infty$.

Proof. Let $v$ be the Lévy measure of $L$. Since $f(x)=f(x)-f_{+}=O(x)$ as $x \downarrow 0$, there exist $\delta>0$ and $M>0$ such that $f(x) \leq M x$ for $x \in(0, \delta]$. Therefore,

$$
\int_{0}^{\delta} x v(\mathrm{~d} x)=\int_{0}^{\delta} \frac{x f(x)}{|x|^{1+\alpha_{+}}} \mathrm{d} x \leq M \int_{0}^{\delta} \frac{x^{2}}{|x|^{1+\alpha_{+}}} \mathrm{d} x<\infty,
$$

showing that the small positive jumps are summable. Of course, the large jumps are always summable.

We now come to the proof of the second part of Theorem 4.2. The main difference to the above is that we cannot use the 1-stable process as a reference since it is not integrable. Instead, we use the normal inverse Gaussian process. It is the symmetry of its Lévy density around 0 that forces us to impose the condition $f_{+}=f_{-}$in the theorem to apply our method. Indeed, we are not aware of a suitable process with sufficiently asymmetric density for which the absolute moment asymptotics are known.

Proof of Theorem 4.2(ii). The proof has the same structure as the proof of part (i).

Step 1: normal inverse Gaussian case. First let $Z$ be a symmetric normal inverse Gaussian process with Lévy measure

$$
v^{\prime}(\mathrm{d} x)=\frac{\rho}{\pi|x|} K_{1}(|x|),
$$

where $\rho>0$ and $K_{\theta}$ denotes the modified Bessel function of the third kind of order $\theta$. Then we are in the setting of Theorem 4.2(ii) with $f_{+}=f_{-}=\rho / \pi$ by the properties of $K_{1}$; see, e.g. [1, Equation (9.6.9)]. The absolute moments of $Z_{t}$ were calculated explicitly and for all $t$ 
by Barndorff-Nielsen and Stelzer [4, Corollary 4]. By their formula and another property of Bessel functions (see [1, Equation (9.6.8)]), we have

$$
\mathrm{E}\left[\left|Z_{T}\right|\right]=\frac{2 \rho}{\pi} \mathrm{e}^{\rho T} T K_{0}(\rho T) \sim \frac{2 \rho}{\pi} T|\log T|=\left(f_{+}+f_{-}\right) T|\log T| .
$$

Step 2: general Lévy case. Now let $Z$ be a $(1,1)$-stable-like Lévy martingale with Lévy measure $v(\mathrm{~d} x)=f(x) /|x|^{2} \mathrm{~d} x$, where, by assumption, $f$ satisfies $f_{0}:=\lim _{x \rightarrow 0} f(x)=$ $f_{+}=f_{-}$. As the $f_{0}=0$ case again follows from Lemma 4.2, we may assume that $f_{0}>0$. Then there exists a small $\delta>0$ such that $f$ is bounded away from 0 on $[-\delta, \delta]$ and we can define

$$
\psi(x):=\mathbf{1}_{[-\delta, \delta]}(x) \frac{f_{0} K_{1}(|x|)|x|}{f(x)} .
$$

By choosing $\rho:=\pi f_{0}$ we have $\nu^{\prime}(\mathrm{d} x)=\psi(x) \nu(\mathrm{d} x)$ on $[-\delta, \delta]$, where $\nu^{\prime}$ is as in step 1 . As $f_{0} K_{1}(|x|)|x|=f_{0}+O(|x|)$ by [1, Equation (9.6.11)], we have

$$
\psi(x)-\mathbf{1}_{[-\delta, \delta]}(x)=\mathbf{1}_{[-\delta, \delta]}(x) \frac{f_{0} K_{1}(|x|)|x|-f(x)}{f(x)}=O(|x|),
$$

owing to the assumption that $f(x)=f_{0}+O(|x|)$. Making $\delta>0$ smaller if necessary, we conclude that $\int_{-\delta}^{\delta}(\psi(x)-1)^{2} v(\mathrm{~d} x)<\infty$, and now the assertion follows from Lemma 4.1 and step 1. The last step to the general martingale case is as in the proof of part (i).

\section{Appendix A}

The following lemma collects some standard facts about Lévy processes that are used throughout the text. A Lévy process $L$ is an adapted càdlàg process with independent and stationary increments and $L_{0}=0$.

Lemma A.1. Let $L$ be a Lévy process having triplet $(b, c, v)$ with respect to the truncation function $h(x)=x \mathbf{1}_{\{|x| \leq 1\}}$.

(i) $\int_{\mathbb{R}} 1 \wedge|x|^{2} v(\mathrm{~d} x)<\infty$.

(ii) There is a decomposition $L_{t}=L_{t}^{a}+L_{t}^{b}+L_{t}^{c}+$ At into independent Lévy processes such that $L^{a}$ is a compound Poisson process, $L^{b}$ is a purely discontinuous martingale with bounded jumps, $L^{c}=\sqrt{c} W$ is a scaled Brownian motion, and $A \in \mathbb{R}$.

(iii) Let $p \in[1, \infty)$. Then $\int_{|x|>1}|x|^{p} v(\mathrm{~d} x)<\infty$ if and only if $\mathrm{E}\left[\left|L_{t}\right|^{p}\right]<\infty$ for all $t \geq 0$. In particular, if $L$ has bounded jumps, or, equivalently, if the support of $v$ is compact, then $\mathrm{E}\left[\left|L_{t}\right|^{p}\right]<\infty$ for all $p \in[1, \infty)$.

(iv) $L$ is a martingale if and only if $\int_{|x|>1}|x| v(\mathrm{~d} x)<\infty$ and $b+\int_{|x|>1} x v(\mathrm{~d} x)=0$ both hold.

(v) If $L$ is integrable then $\mathrm{E}\left[L_{t}\right]=t \mathrm{E}\left[L_{1}\right]$ and $L_{t}-t \mathrm{E}\left[L_{1}\right]$ is a martingale.

(vi) If $L$ is a square-integrable martingale then

$$
\mathrm{E}\left[L_{t}^{2}\right]=\mathrm{E}\left[[L, L]_{t}\right]=\langle L, L\rangle_{t}=t\langle L, L\rangle_{1}=t c+t \int_{\mathbb{R}}|x|^{2} v(\mathrm{~d} x)<\infty .
$$


(vii) If $c=0, L$ is of finite variation if and only if $\int_{|x| \leq 1}|x| \nu(\mathrm{d} x)<\infty$ and of integrable variation if and only if $\int_{\mathbb{R}}|x| v(\mathrm{~d} x)<\infty$. In that case the total variation $\operatorname{var}(L)_{t}:=$ $\int_{0}^{t}\left|\mathrm{~d} L_{s}\right|$ is a Lévy process satisfying $\mathrm{E}\left[\operatorname{var}(L)_{1}\right]=\int|x| v(\mathrm{~d} x)+\left|\int x v(\mathrm{~d} x)\right|$.

(viii) For any $\delta>0$, there is a decomposition $L=L^{\leq \delta}+L^{>\delta}$ into two independent Lévy processes satisfying $\left|\Delta L^{\leq \delta}\right| \leq \delta$ as well as $\left|\Delta L^{>\delta}\right|>\delta$ on the set $\left\{\left|\Delta L^{>\delta}\right|\right\}>0$. The corresponding Lévy measures are given by $v^{\leq \delta}(\mathrm{d} x)=\mathbf{1}_{[-\delta, \delta]}(x) v(\mathrm{~d} x)$ and $v^{>\delta}(\mathrm{d} x)=$ $\mathbf{1}_{\mathbb{R} \backslash[-\delta, \delta]}(x) \cup(\mathrm{d} x)$. If $L^{>\delta}$ has no Brownian component, it is a compound Poisson process with drift.

(ix) If $L$ is a martingale, the stochastic exponential $\mathcal{E}(L)$ is again a martingale.

(x) If $L$ is a square-integrable martingale then so is $\mathcal{E}(L)$ and, moreover, $\mathrm{E}\left[\mathcal{E}(L)_{t}^{2}\right]=$ $\exp \left(t\langle L, L\rangle_{1}\right)$.

Proof. Statements (i)-(viii) can be found in any advanced textbook about Lévy processes; see, e.g. [26]. Statement (ix) is [8, Proposition 8.23]. One way to deduce the formula in (x) is to use Yor's formula in

$$
\begin{aligned}
\mathcal{E}(L)_{t}^{2} & =\mathcal{E}(2 L+[L, L]-\langle L, L\rangle+\langle L, L\rangle)_{t} \\
& =\mathcal{E}(2 L+[L, L]-\langle L, L\rangle)_{t} \exp \left(t\langle L, L\rangle_{1}\right) .
\end{aligned}
$$

Noting that $2 L+[L, L]-\langle L, L\rangle$ is a Lévy martingale, (ix) yields the result.

The following makes precise a remark from the introduction.

Remark A.1. Let $S$ be any càdlàg martingale with absolutely continuous predictable characteristics. Then $S$ can be represented in the form (1.2). Indeed, let

$$
\mathrm{d} B_{t}=b_{t} \mathrm{~d} t, \quad \mathrm{~d} C_{t}=\sigma_{t}^{2} \mathrm{~d} t, \quad \mathrm{~d} v_{t}=K_{t}(\mathrm{~d} x) \mathrm{d} t
$$

be the characteristics of $S$ with respect to the trivial truncation function $h(x)=x$ (cf. [20, Chapter II] for background). The latter choice is possible since $S$ is a martingale, which then implies that $B=0$. Moreover, let $F$ be any atomless $\sigma$-finite measure on $\mathbb{R}$ such that $F(\mathbb{R})=\infty$. Then there exist a Brownian motion $W$ and a Poisson random measure $N$ with compensator $F(\mathrm{~d} x) \mathrm{d} t$ such that (1.2) holds. Moreover, $\kappa$ and $K$ satisfy the relation $K_{t}(A)=F\left(\kappa_{t}^{-1}(A)\right)$ for any Borel set $A$, where $\kappa_{t}^{-1}$ denotes the preimage with respect to the spatial variable $x$. To be precise, the constructions of $W$ and $N$ may necessitate an enlargement of the probability space, but this is harmless since we are interested only in distributional properties. We refer the reader to [18, Theorem 14.68(a)] for further details.

\section{Acknowledgements}

We thank Martin Keller-Ressel, Sergey Nadtochiy, and Mark Podolskij for discussions, and an anonymous referee for detailed comments. The first author was partially supported by the National Centre of Competence in Research 'Financial Valuation and Risk Management' (NCCR FINRISK), Project D1 (Mathematical Methods in Financial Risk Management), of the Swiss National Science Foundation. The second author acknowledges financial support from the Swiss National Science Foundation, under grant PDFM2-120424/1. 


\section{References}

[1] Abramowitz, M. and Stegun, I. A. (1964). Handbook of Mathematical Functions with Formulas, Graphs, and Mathematical Tables. US Government Printing Office, Washington, DC.

[2] Alòs, E., LÉON, J. A. AND VIVES, J. (2007). On the short-time behavior of the implied volatility for jump-diffusion models with stochastic volatility. Finance Stoch. 11, 571-589.

[3] Andersen, L. B. G. and Piterbarg, V. V. (2007). Moment explosions in stochastic volatility models. Finance Stoch. 11, 29-50.

[4] BarndorfF-Nielsen, O. E. ANd Stelzer, R. (2005). Absolute moments of generalized hyperbolic distributions and approximate scaling of normal inverse Gaussian Lévy processes. Scand. J. Statist. 32, 617-637.

[5] Berestycki, H., Busca, J. and Florent, I. (2002). Asymptotics and calibration of local volatility models. Quant. Finance 2, 61-69.

[6] Berestycki, H., Busca, J. AND Florent, I. (2004). Computing the implied volatility in stochastic volatility models. Commun. Pure Appl. Math. 57, 1352-1373.

[7] Carr, P. and Wu, L. (2003). What type of process underlies options? A simple robust test. J. Finance 58, 2581-2610.

[8] Cont, R. And Tankov, P. (2004). Financial Modelling with Jump Processes. Chapman \& Hall/CRC, Boca Raton, FL.

[9] Cox, J. C., Ingersoll, J. E., Jr. And Ross, S. A. (1985). A theory of the term structure of interest rates. Econometrica 53, 385-407.

[10] Durrleman, V. (2008). Convergence of at-the-money implied volatilities to the spot volatility. J. Appl. Prob. 45, 542-550.

[11] Durrleman, V. (2010). From implied to spot volatilities. Finance Stoch. 14, 157-177.

[12] Feng, J., Forde, M. ANd Fouque, J.-P. (2010). Short-maturity asymptotics for a fast mean-reverting Heston stochastic volatility model. SIAM J. Financial Math. 1, 126-141.

[13] Figueroa-López, J. E. AND Forde, M. (2011). The small-maturity smile for exponential Lévy models. To appear in SIAM J. Financial Math. Available at http://arxiv.org/abs/1105.3180v1.

[14] Forde, M. (2009). Small-time asymptotics for a general local-stochastic volatility model, using the heat kernel expansion. Preprint.

[15] Forde, M. AND Jacquier, A. (2009). Small-time asymptotics for implied volatility under the Heston model. Internat. J. Theoret. Appl. Finance 12, 861-876.

[16] Forde, M., JACQuier, A. ANd LeE, R. (2011). The small-time smile and term structure of implied volatility under the Heston model. Preprint.

[17] Gatheral, J., Hsu, E. P., Laurence, P. M., Ouyang, C. and Wang, T.-H. (2011). Asymptotics of implied volatility in local volatility models. To appear in Math. Finance.

[18] JACOD, J. (1979). Calcul Stochastique et Problèmes de Martingales (Lecture Notes Math. 714). Springer, Berlin.

[19] JACOD, J. (2007). Asymptotic properties of power variations of Lévy processes. ESAIM Prob. Statist. 11, $173-196$.

[20] Jacod, J. ANd Shiryaev, A. N. (2003). Limit Theorems for Stochastic Processes, 2nd edn. Springer, Berlin.

[21] Luschgy, H. and Pagès, G. (2008). Moment estimates for Lévy processes. Electron. Commun. Prob. 13, 422-434.

[22] Medvedev, A. And Scaillet, O. (2007). Approximation and calibration of short-term implied volatilities under jump-diffusion stochastic volatility. Rev. Financial Studies 20, 427-459.

[23] Protter, P. E. (2005). Stochastic Integration and Differential Equations, 2nd edn. Springer, Berlin.

[24] Roper, M. (2008). Implied volatility explosions: European calls and implied volatilities close to expiry in exponential Lévy models. Preprint.

[25] Samorodnitsky, G. And Taqqu, M. S. (1994). Stable Non-Gaussian Random Processes. Chapman \& Hall, New York.

[26] Sato, K.-I. (1999). Lévy Processes and Infinitely Divisible Distributions. Cambridge University Press.

[27] Tankov, P. (2010). Pricing and hedging in exponential Lévy models: Review of recent results. In Paris-Princeton Lectures on Mathematical Finance 2010 (Lecture Notes Math. 2003), Springer, Berlin, pp. 319-359. 